\title{
On a Non-Linear Semi-Group Attached to Stochastic Optimal Control
}

\author{
By
}

\author{
Makiko Nisı**
}

\section{§ 1. Introduction}

In [6] we introduced a non-linear semi-group attached to the stochastic control of diffusion type, by the following way. Let $\Gamma$ be a $\sigma$-compact subset of $\boldsymbol{R}^{k}$, called by a control region. Let a triple $(\Omega, B, U)$ be an admissible system where $\Omega$ is a probability space, $B$ is an $n$-dimensional Brownian motion on $\Omega$ and $U$ is a $\Gamma$-valued $B$-non-anticipative process on $\Omega$. For an admissible system $(\Omega, B, U)$ we consider the following $n$-dimensional stochastic differential equation

$$
d X(t)=\alpha(X(t), U(t)) d B(t)+\gamma(X(t), U(t)) d t
$$

where $\alpha(x, u)$ is a symmetric $n \times n$-matrix and $\gamma(x, u)$ an $n$-vector. Under the condition of smoothness and boundness of the coefficients $\alpha$ and $\gamma$, there exists a unique solution $X$, which is called the response for $U$.

By $C$ we denote the Banach lattice of all bounded and uniformly continuous functions on $\mathbb{R}^{n}$ endowed with the usual supremum norm and the usual order. Let $c(x, u)$ be non-negative and $f(x, u)$ real. We assume that both $c$ and $f$ are smooth and bounded. For any $\phi \in C$ we define $Q_{t}$ by

$$
\begin{aligned}
& Q_{t} \phi(x)=\sup _{\text {adm. syst. }} E_{x} \int_{0}^{\iota} \exp \left\{-\int_{0}^{s} c(X(\theta), U(\theta)) d \theta\right\} \\
& \quad \times f(X(s), U(s)) d s+\exp \left\{-\int_{0}^{\iota} c(X(\theta), U(\theta)) d \theta\right\} \phi(X(t)),
\end{aligned}
$$

where $X$ is the response for $U$, starting at $X(0)=x$. Then $Q_{t}$ is a strongly continuous non-linear semi-group on $C$, which is contractive and

Communicated by K. Itô, May 10, 1976.

* Department of Mathematics, Kobe University, Kobe 657, Japan. 
monotone. Moreover the generator $G$ of $Q_{t}$ is given by

$$
\begin{gathered}
G \phi=\sup _{u \in \Gamma}\left[A^{u} \phi+f^{u}\right] \\
A^{u} \phi(x)=\frac{1}{2} \sum_{i j} \alpha^{2}(x, u)_{i j} \frac{\partial^{2} \phi}{\partial x_{i} \partial x_{j}}(x) \\
+\sum_{i} \gamma_{i}(x, u) \frac{\partial \phi}{\partial x_{i}}(x)-c(x, u) \phi(x)
\end{gathered}
$$

for $\phi$ whose first and second derivatives are in $C$. The right side of (3) can be found in the famous Bellman equation, [2], [4]. Furthermore the least $Q_{t}$-excessive majorant has a close relation to the optimal stopping problem, [3], [4].

In this note we shall discuss a similar problem in a more general set-up. Let $A^{u}$ be the generator of a Markov process. We seek a semigroup of operators acting on $L_{\infty}\left(\mathbb{R}^{n}, \mu\right)$ whose generator is an extension of $G \phi=\sup _{u}\left(A^{u} \phi+f^{u}\right)$. Such a semi-group (with generator $G$ ) will be obtained as the envelope of the semi-groups

$$
T_{t}^{u} \phi=P_{t}^{u} \phi+\int_{0}^{t} P_{\theta}^{u} f^{u} d \theta \quad u \in \Gamma
$$

whose generators are

$$
G^{u} \phi=A^{u} \phi+f^{u}, \quad u \in \Gamma
$$

respectively, as we can image from the fact that $G$ is the envelope of $G^{u}, u \in \Gamma$. In fact we will prove the following theorem in $\S 3$.

Theorem 1. Let $A^{u}$ be the generator of positive contractive and strongly continuous linear semi-group $P_{t}^{u}$ on $L_{\infty}\left(\mathbb{R}^{n}, \mu\right)$. We assume the following conditions (A1) (A3).

(A1) If $\phi_{n} \in L_{\infty}\left(\mathbb{R}^{n}, \mu\right)$ is an increasing sequence tending to $\phi \in L_{\infty}$ $\left(\mathbb{R}^{n}, \mu\right) \mu$-a.e., then $P_{t}{ }^{u} \phi_{n}$ increases and tends to $P_{t}^{u} \phi \mu$-a.e. for every $u \in \Gamma$ and every $t \geq 0$.

(A2) Let $D\left(A^{u}\right)$ denote the domain of the generator $A^{u}$. The subset $D$ of $L_{\infty}\left(\mathbb{R}^{n}, \mu\right)$ defined by

$$
D=\left\{\phi \in \bigcap_{u} D\left(A^{u}\right) ; \sup _{u}\left\|A^{u} \phi\right\|<\infty\right\}
$$


Non-Linear Semi-Group Attached to Stochastic Optimal Control 515 is strongly dense in $L_{\infty}\left(\boldsymbol{R}^{n}, \mu\right)$.

$$
\sup _{u}\left\|f^{u}\right\|<\infty .
$$

Then there exists a unique non-linear semi-group $S_{t}$ on $L_{c}\left(\mathbb{R}^{n}, \mu\right)$ satisfying the following conditions $(0) \sim(v i)$ :

(0) semi-group property: $S_{0}=$ identity, $S_{t+\theta} \phi=S_{t}\left(S_{\theta} \phi\right)=S_{\theta}\left(S_{t} \phi\right)$,

(i) monotone: $S_{t} \phi \leq S_{t} \psi$, whenever $\phi \leq \psi$,

(ii) contractive: $\left\|S_{t} \phi-S_{t} \psi\right\| \leq\|\phi-\psi\|$,

(iii) strongly continuous: $\left\|S_{t} \phi-S_{\theta} \phi\right\| \rightarrow 0$, as $t \rightarrow \theta$,

(iv) $P_{t}^{u} \phi+\int_{0}^{t} P_{\theta}^{u} f^{u} d \theta \leq S_{t} \phi$, for $\forall t$ and $u$, where the integral stands for the Bochner integral,

(v) the generator $G$ of $S_{t}$ is expressed by

$$
G \phi=\sup _{u}\left[A^{u} \phi+f^{u}\right] \text { for } \phi \in D(G) \cap D,
$$

(vi) minimum: if $\widetilde{S}_{t}$ is a non-linear semi-group with (i) $\sim(\mathrm{iv})$, then

$$
S_{t} \phi \leq \widetilde{S}_{t} \phi
$$

In $\S 4$, we shall show the existence of the least $S_{t}$-excessive function.

Theorem 2. Suppose that there exists a positive $c$ such that $\left|P_{t}{ }^{u}\right| \leq e^{-c t}$ for any $u$. Then, for any $g \in L_{\infty}\left(\mathbb{R}^{n}, \mu\right)$, there exists a unique $v \in L_{\infty}\left(\boldsymbol{R}^{n}, \mu\right)$ such that

(i) $S_{t}$-excessive majorant of $g: \quad g \leq v$ and $S_{t} v \leq v \quad \forall t \geq 0$

(ii) least: if $V$ is an $S_{t}$-excessive majorant of $g$, then $v \leq V$.

In $\S 5$ we will mention two simple examples as applications of our results. Since we formulate control problems in terms of non-linear semigroups on $L_{\infty}\left(\boldsymbol{R}^{n}, \mu\right)$ in this note, the stochastic control of diffusion type does not lie in our framework, but some optimal controls can be treated in our way, as we shall see in $\S 5$.

The author wishes to express her sincere thanks to Professors K. Itô and S. Watanabe for their valuable suggestions. 


\section{$\S 2 . \quad$ Preliminaries}

Let $\mu$ be a $\sigma$-finite measure on $\boldsymbol{R}^{n}$. Let $L\left(\equiv L_{\infty}\left(\boldsymbol{R}^{n}, \mu\right)\right)$ denote the set of all Borel measurable, essential bounded functions, defined $\mu$-a.e. on $\mathbb{R}^{n}$. $L$ becomes a complete Banach lattice by the usual norm and partial order, [cf. 7], i.e.

$$
\|\phi\| \equiv \underset{x \in \boldsymbol{R}^{n}}{\operatorname{ess.sup}}|\phi(x)|
$$

and " $\phi \leq \psi$ " is defined by " $\phi(x) \leq \psi(x), \mu$-a.e." A subset $\left\{\phi_{\alpha}\right\}$ of $L$ is said to be $O$-bounded, if there exist $\underline{\phi}$ and $\bar{\phi}$ in $L$ such that

$$
\underline{\phi} \leq \psi_{\alpha} \leq \bar{\psi}, \quad \forall \alpha .
$$

Hence a subset $\left\{\psi_{\alpha}\right\}$ of $L$ is $O$-bounded, if and only if " $\sup _{\alpha}\left\|\psi_{\alpha}\right\|<\infty$ ". When $\psi_{n} \in L$ increasingly tends to $\psi \in L$, we say $\psi=O_{i}-\lim _{n} \psi_{n}$. Hence, if $\psi=O_{i}-\lim \psi_{n}$, then " $\sup _{u}\left\|\psi_{n}\right\|<\infty$ ". In this note we often use the following well-known facts,

Proposition 1. For any O-bounded set $\left\{\psi_{\alpha}\right\}$ of $L$ there uniquely exist $\psi^{+}$and $\psi^{-}$in $L$ such that

(i) $\psi_{\alpha} \leq \psi^{+}, \forall \alpha$

(ii) if $\psi$ satisfies " $\psi_{\alpha} \leq \psi, \forall \alpha$ ", then $\psi^{+} \leq \psi$,

and

(i) ${ }^{\prime} \psi^{-} \leq \psi_{\alpha}, \quad \forall \alpha$

(ii)' if $\psi$ satisfies " $\psi \leq \psi_{\alpha}, \forall \alpha$ ", then $\psi \leq \psi^{-}$, $\sup \psi_{\alpha}$ and $\inf \psi_{\alpha}$ are denoted by $\psi^{+}$and $\psi^{-}$respectively.

Moreover,

$$
\begin{gathered}
\inf \left(\phi_{\alpha}-\psi_{\alpha}\right) \leq \sup \phi_{\alpha}-\sup \psi_{\alpha} \leq \sup \left(\phi_{\alpha}-\psi_{\alpha}\right) . \\
\left\|\sup \phi_{\alpha}-\sup \psi_{\alpha}\right\| \leq \sup \left\|\phi_{\alpha}-\psi_{\alpha}\right\| .
\end{gathered}
$$

Let $T_{t} \phi$ be strongly continuous in $t$. Then $T_{t} \phi$ has a $(t, x)$-Borel measurable version which is continuous in $t$.

Proof. Let $\left\{r_{i}\right\}$ be countable and dense in $[0, \infty)$ and $\Phi\left(r_{i}, \cdot\right)$ a Borel measurable version of $T_{r_{i}} \phi$. Then the set $\Sigma$ of $\left\{x \in \mathbb{R}^{n} ; \mid \Phi\left(r_{i}, x\right)\right.$ $\left.-\Phi\left(r_{j}, x\right) \mid \leq\left\|T_{r_{i}} \phi-T_{r_{i}} \phi\right\| \forall i j\right\}$ is $\mu$-full. On the other hand, for any positives $\varepsilon$ and $l$, there exists a positive $\delta$ such that 
Non-Linear Semi-Group Attached to Stochastic Optimal Control 517 $\left\|T_{t} \phi-T_{\theta} \phi\right\|<\varepsilon \quad$ whenever $\quad|t-\theta|<\delta$ and $0 \leq t, \theta \leq l$.

Hence, for $x \in \Sigma, \Phi\left(r_{i}, x\right)$ is uniformly continuous on $\left\{r_{i}\right\} \subset[0, l]$. Thus, $\Phi(\cdot, x)$ can be extended to a continuous function $\widetilde{\Phi}(\cdot, x)$ on $[0, l]$. Letting $l$ tend to $\infty$, we get our wanted version $\widetilde{\emptyset}$.

The Bochner integral $\int_{0}^{t} T_{\theta} \phi d \theta$ can understood as the usual Rieman integral $\int_{0}^{t} \widetilde{\Phi}(\theta, x) d \theta$.

Let $P_{t}$ be a positive, contractive and strongly continuous linear semigroup on $L$. Define $T_{t}$ for $f \in L$ by

$$
T_{t} \phi=P_{t} \phi+\int_{0}^{t} P_{\theta} f d \theta, \quad \phi \in L .
$$

Then $T_{t}$ is a mapping from $L$ into $L$ and has the following properties (T0) semi-group property: $T_{0} \phi=\phi, T_{t+\theta} \phi=T_{t}\left(T_{\theta} \phi\right)=T_{\theta}\left(T_{t} \phi\right)$,

(T1) monotone: $T_{t} \phi \leq T_{t} \psi$ whenever $\phi \leq \psi$,

(T2) contractive; $\left\|T_{t} \phi-T_{t} \psi\right\| \leq\|\phi-\phi\|$

(T3) strongly continuous: $\left\|T_{t} \phi-T_{\theta} \phi\right\| \rightarrow 0$ as $t \rightarrow \theta$

(T4) the generator $G$ of $T_{t}$ : Let $A$ be the generator of $P_{t}$. Then $D(G)=D(A)$ and

$$
\begin{aligned}
G \phi & =A \phi+f \\
T_{t} \phi-\phi & =\int_{0}^{t} P_{\theta} G \phi d \theta \quad \forall \phi \in D(G) .
\end{aligned}
$$

Proof. Since (T1), (T2) and (T3) are obvious, we shall only show (T0), (T4) and (T5).

$$
\begin{aligned}
& \text { (T0). } T_{t+\theta} \phi=P_{t+\theta} \phi+\int_{0}^{t+\theta} P_{s} f d s=P_{\theta}\left(P_{t} \phi\right)+\int_{\theta}^{t+\theta} P_{s} f d s+\int_{0}^{\theta} P_{s} f d s \\
& =P_{\theta}\left(P_{t} \phi+\int_{0}^{t} P_{s} f d s\right)+\int_{0}^{\theta} P_{s} f d s=P_{\theta}\left(T_{t} \phi\right)+\int_{0}^{\theta} P_{s} f d s=T_{\theta}\left(T_{t} \phi\right) .
\end{aligned}
$$

(T4). For $\varepsilon>0$, there exists a positive $\delta$ such that $\left\|P_{\theta} f-f\right\|<\varepsilon$ for $\theta<\delta$. Hence

$$
\begin{aligned}
&\left\|\frac{1}{t} \int_{0}^{t} P_{\theta} f d \theta-f\right\|=\left\|\frac{1}{t} \int_{0}^{t}\left(P_{\theta} f-f\right) d \theta\right\| \\
& \leq \frac{1}{t} \int_{0}^{t}\left\|P_{\theta} f-f\right\| d \theta<\varepsilon \text { for } t<\delta .
\end{aligned}
$$


Therefore $\lim _{t \downarrow 0} \frac{1}{t}\left(T_{t} \phi-\phi\right)$ exists if and only if $\lim _{t \downarrow 0} \frac{1}{t}\left(P_{t} \phi-\phi\right)$ exists. Moreover (2) is valid.

(T5). For any $\phi \in D(A)$, we have

$$
\begin{aligned}
T_{t} \phi-\phi & =P_{t} \phi-\phi+\int_{0}^{t} P_{\theta} f d \theta \\
& =\int_{0}^{t} P_{\theta} A \phi d \theta+\int_{0}^{t} P_{\theta} f d \theta=\int_{0}^{t} P_{\theta}(A \phi+f) d \theta .
\end{aligned}
$$

Proposition 2. Suppose (A1) and (A3). If $\phi=O_{i}-\lim \phi_{n}$ then

$$
\sup _{u} T_{t}^{u} \phi=O_{i}-\lim _{n} \sup _{u} T_{t}{ }^{u} \phi_{n}
$$

Proof. Since $T_{t}{ }^{u}$ satisfies (T1) and (T2), we have $T_{t}{ }^{u} \phi_{n} \leq T_{t}{ }^{u} \phi_{n+1}$ and

$$
\left\|T_{t}{ }^{u} \phi_{n}\right\| \leq\left\|T_{t}{ }^{u} \phi_{n}-T_{t}{ }^{u} O\right\|+\left\|T_{t}{ }^{u} O\right\| \leq\left\|\phi_{n}\right\|+\sup \left\|f^{u}\right\| t .
$$

Thus $\sup _{n} T_{t}{ }^{u} \phi_{n}$ is increasing as $n \rightarrow \infty$ and the set $\left\{\sup _{u} T_{t}{ }^{u} \phi_{n}, n=1,2, \cdots\right\}$ is $O$-bounded. Therefore

$$
O_{i}-\lim _{n} \sup _{u} T_{t}^{u} \phi_{n} \leq \sup _{u} T_{t}^{u} \phi .
$$

On the other hand, from (A1) we can derive, for any $u$

$$
T_{t}^{u} \phi=O_{i}-\lim _{n} T_{t}^{u} \phi_{n} \leq O_{i}-\lim _{n} \sup _{u} T_{t}^{u} \phi_{n}
$$

By (5) and (6) we conclude Proposition 2.

\section{$\S 3$. Proof of Theorem $\mathbb{1}$}

We shall construct our required semi-group $S_{t}$. Define $J=J(N)$ by

$$
J \phi=\sup _{u} T_{1 / 2^{N}}^{u} \phi, \phi \in L .
$$

Then $J$ is a mapping from $L$ into $L$. Define $J^{k}$ by

$$
J^{k+1} \phi=J\left(J^{k} \phi\right) \text { and } J^{0} \phi=\phi .
$$

Lemma 1. $J^{k}$ has the following properties,

(J0) $J^{k+l} \phi=J^{k}\left(J^{l} \phi\right)=J^{l}\left(J^{k} \phi\right)$,

(J1) monotone: $J^{k} \phi \leq J^{k} \psi$ whenever $\phi \leq \psi$, 
Non-Linear Semi-Group Attached to Stochastic Optimal Control 519

(J2) contractive: $\left\|J^{k} \phi-J^{k} \psi\right\| \leq\|\phi-\psi\|$

$$
\left\|J^{k} \phi-\phi\right\| \leq \frac{k}{2^{N}}\left(\sup _{u}\left\|A^{u} \phi\right\|+\sup _{u}\left\|f^{u}\right\|\right) \quad \text { for } \quad \phi \in D,
$$

$$
T_{k / 2 \alpha}^{u} \phi \leq J^{k} \phi
$$

$$
J^{k} \phi=O_{i}-\lim _{n} J^{k} \phi_{n} \quad \text { if } \quad \phi=O_{i}-\lim _{n} \phi_{n} .
$$

Proof. Since $T_{t}{ }^{u}$ is monotone, we have

$$
J \phi \leq J \psi \text { whenever } \phi \leq \psi \text {. }
$$

Hence we can show (J1) by induction.

Put $\Delta=\frac{1}{2^{N}}$. The following evaluation is clear,

$$
\|J \phi-J \psi\|=\left\|\sup _{u} T_{\Delta}^{u} \phi-\sup _{u} T_{\Delta}^{u} \psi\right\| \leq \sup _{u}\left\|T_{\Delta}^{u} \phi-T_{\Delta}{ }^{u} \psi\right\| \leq\|\phi-\psi\| .
$$

Thus if we assume that (J2) holds for $k$, then

$$
\left\|J^{k+1} \phi-J^{k+1} \psi\right\|=\left\|J\left(J^{k} \phi\right)-J\left(J^{k} \psi\right)\right\| \leq\left\|J^{k} \phi-J^{k} \psi\right\| \leq\|\phi-\psi\|
$$

namely (J2) holds for $k+1$.

Put $K(\phi)=\sup _{u}\left\|A^{u} \phi\right\|+\sup _{u}\left\|f^{u}\right\|$. Recalling (T5) we have, for $\phi \in D$

$$
T_{\Delta}^{u} \phi-\phi=\int_{0}^{\Delta} P_{\theta}^{u} A^{u} \phi d \theta+\int_{0}^{\Delta} P_{\theta}^{u} f^{u} d \theta \text {. }
$$

So

$$
\|J \phi-\phi\| \leq \sup _{u}\left\|T_{\Delta}{ }^{u} \phi-\phi\right\| \leq \Delta K(\phi) .
$$

Therefore by (J2) we see

$$
\begin{gathered}
\left\|J^{k} \phi-\phi\right\| \leq \sum_{j=1}^{k}\left\|J^{j} \phi-J^{j-1} \phi\right\|=\sum_{j=1}^{k}\left\|J^{j-1}(J \phi)-J^{j-1} \phi\right\| \\
\leq k\|J \phi-\phi\| \leq k \Delta \cdot K(\phi) .
\end{gathered}
$$

This completes the proof of (J3).

By the definition of $J$ we get

$$
T_{\Delta}{ }^{u} \psi \leq J \psi \quad \forall \psi \in L .
$$

Hence, if we assume that (J4) holds for $k$, then

$$
T_{(k+1) \Delta}^{u} \phi=T_{\Delta}{ }^{u}\left(T_{k \Delta}^{u} \phi\right) \leq T_{\Delta}{ }^{u}\left(J^{k} \phi\right) \leq J\left(J^{k} \phi\right)=J^{k+1} \phi,
$$


namely (J4) holds for $k+1$.

For $k=1$, (J5) is Proposition 2 in $\S 2$. If (J5) holds for $k$, then $J^{k+1} \phi=J\left(J^{k} \phi\right)=J\left(O_{i}-\lim J^{k} \phi_{n}\right)=O_{i}-\lim J\left(J^{k} \phi_{n}\right)=O_{i}-\lim J^{k+1} \phi_{n}$.

Therefore we get (J5).

Put $S_{t}^{(N)} \phi=J^{k}(N) \phi$ for $t=\frac{k}{2^{N}}, k=0,1,2, \cdots$.

Lemma 2. $S_{t}{ }^{(N)}$ is increasing as $N \rightarrow \infty$, i.e.

$$
S_{t}^{(N)} \phi \leq S_{t}^{(N+1)} \phi \quad \text { for } \quad t=\frac{k}{2^{N}}
$$

Proof. Put $\Delta=1 / 2^{N+1}$. Recalling (T0) and (T1), we have

$$
T_{2 \Delta}^{u} \phi=T_{\Delta}{ }^{u}\left(T_{\Delta}{ }^{u} \phi\right) \leq T_{\Delta}{ }^{u}\left(S_{\Delta}{ }^{(N+1)} \phi\right) .
$$

Taking the supremum of both sides, we get

$$
S_{2 \Delta}^{(N)} \phi \leq S_{\Delta}^{(N+1)}\left(S_{\Delta}{ }^{(N+1)} \phi\right)=S_{2 \Delta}^{(N+1)} \phi,
$$

namely (2) is valid for $k=1$. If (2) holds for $k$, then

$$
\begin{aligned}
S_{2(k+1) \Delta}^{(N)} \phi= & S_{2 \Delta}^{(N)}\left(S_{2 k \Delta}^{(N)} \phi\right) \leq S_{2 \Delta}^{(N)}\left(S_{2 k \Delta}^{(N+1)} \phi\right) \\
& \leq S_{2 \Delta}^{(N+1)}\left(S_{2 k \Delta}^{(N+1)} \phi\right)=S_{2(k+1) \Delta}^{(N+1)} \phi
\end{aligned}
$$

This completes the proof of Lemma 2 .

Hereafter we put $h=\sup _{u}\left\|f^{u}\right\|$. By virtue of (J2), putting $\Delta=\frac{1}{2^{N}}$ and $t=k \Delta$ we have

$$
\left\|S_{t}^{(N)} \phi\right\| \leq\left\|S_{t}^{(N)} \phi-S_{t}^{(N)} O\right\|+\left\|S_{t}^{(N)} O\right\| \leq\|\phi\|+\left\|S_{t}^{(N)} O\right\|
$$

and

$$
\left\|S_{\Delta}{ }^{(N)} O\right\| \leq \sup _{u}\left\|\int_{0}^{\Delta} P_{\theta}^{u} f^{u} d \theta\right\| \leq \Delta h
$$

Suppose $\left\|S_{k \Delta}^{(N)} O\right\| \leq k \Delta h$. Then

$$
\begin{aligned}
\left\|S_{(k+1) \Delta}^{(N)} O\right\|= & \left\|S_{\Delta}^{(N)}\left(S_{k \Delta}^{(N)} O\right)\right\| \leq \sup _{u}\left\|T_{\Delta}{ }^{u}\left(S_{k \Delta}^{(N)} O\right)\right\| \\
& \leq\left\|S_{k \Delta}^{(N)} O\right\|+\Delta h \leq(k+1) \Delta h
\end{aligned}
$$

Hence we have 
Non-Linear Semi-Group Attached to Stochastic Optimal Control 521

$$
\left\|S_{t}^{(N)} \phi\right\| \leq\|\phi\|+t h
$$

This implies that, for any fixed binary $t=\frac{j}{2^{l}}$, the set $\left\{S_{t}^{(N)} \phi, N \geq l\right\}$ is $O$-bounded. So we can define $S_{t}$ by

$$
S_{t} \phi=O_{i}-\lim _{n} S_{t}^{(n)} \phi \quad \text { for binary } t .
$$

$S_{t}$ has the following properties:

Lemma 3. For binary $t$ and $\theta$, (S0) $S_{0} \phi=\phi$,

(S1) monotone: $S_{t} \phi \leq S_{t} \psi$, whenever $\phi \leq \psi$,

(S2) contractive: $\left\|S_{t} \phi-S_{t} \psi\right\| \leq\|\phi-\psi\|$

(S3) $\left\|S_{t} \phi-S_{\theta} \psi\right\| \leq|t-\theta| K(\phi)$ for $\phi \in D$,

(S4) $\quad T_{t}{ }^{u} \phi \leq S_{t} \phi$.

Proof. From the definition of $S_{t}$ and Lemma 1, these properties are clear. We shall only show (S3). Put $t=\frac{i}{2^{l}}$ and $\theta=\frac{j}{2^{l}},(j \leq i)$. For any $N \geq l$, we have

$$
\left\|S_{t}{ }^{(N)} \phi-S_{\theta}{ }^{(N)} \phi\right\|=\left\|S_{\theta}{ }^{(N)}\left(S_{t-\theta}^{(N)} \phi\right)-S_{\theta}{ }^{(N)} \phi\right\| \leq\left\|S_{t-\theta}^{(N)} \phi-\phi\right\| \leq|t-\theta| K(\phi) .
$$

Since $S_{t}{ }^{(N)} \phi-S_{\theta}{ }^{(N)} \phi$ converges to $S_{t} \phi-S_{\theta} \phi \mu-$ a.e. as $N \rightarrow \infty$, we get

$$
\left\|S_{t} \phi-S_{\theta} \phi\right\| \leq \lim _{N \rightarrow \infty}\left\|S_{t}^{(N)} \phi-S_{\theta}{ }^{(N)} \phi\right\| \leq|t-\theta| K(\phi) .
$$

Using (S3) we can define $S_{t} \phi, t \geq 0$, by

$$
S_{t} \phi=\lim S_{t_{l}} \phi, \phi \in D,
$$

where $\left\{t_{l}\right\}$ is a sequence of binary times approximating $t$. (S3) implies that the left side of (10) does not depend on the special choice of $\left\{t_{l}\right\}$. Moreover $(\mathrm{S} 1) \sim(\mathrm{S} 4)$ hold.

Lemma $3^{\prime}$. For $\theta, t \geq 0$ and $\psi, \phi \in D$,

(S1)' monotone: $S_{t} \phi \leq S_{t} \psi$ whenever $\phi \leq \psi$,

$(\mathrm{S} 2)^{\prime}$ contractive: $\left\|S_{t} \phi-S_{t} \psi\right\| \leq\|\phi-\psi\|$,

$(\mathrm{S} 3)^{\prime} \quad\left\|S_{t} \phi-S_{\theta} \phi\right\| \leq ! t-\theta \mid K(\phi)$,

(S4)' $T_{t}{ }^{u} \phi \leq S_{t} \phi$. 
Recalling (A2) and (S2)', we can extend $S_{t}$ on $L$ by

$$
S_{t} \phi=\lim S_{t} \phi_{n}, \phi \in L,
$$

where $\left\{\phi_{n}\right\}$ is a sequence of functions in $D$ approximating $\phi$.

Proposition 3. $S_{t}$ has the following properties

(i) monotone: $S_{t} \phi \leq S_{t} \psi$ whenever $\phi \leq \psi$,

(ii) contractive: $\left\|S_{t} \phi-S_{t} \psi\right\| \leq\|\phi-\psi\|$,

(iii) strongly continuous: $\left\|S_{t} \phi-S_{\theta} \phi\right\| \rightarrow 0$ as $t \rightarrow \theta$,

(iv) $T_{t}^{u} \phi \leq S_{t} \phi$.

Proof. First we shall show (ii). Take $\phi_{n} \in D$ and $\psi_{n} \in D$ approximating $\phi$ and $\psi$ respectively. Hence

$$
\left\|S_{t} \phi-S_{t} \psi\right\| \leq \lim _{n}\left\|S_{t} \phi_{n}-S_{t} \psi_{n}\right\| \leq \lim _{n}\left\|\phi_{n}-\psi_{n}\right\|=\|\phi-\psi\| .
$$

(i). For $\varepsilon>0$, we take an approximation $\phi_{n}(\varepsilon) \in D$ to $\phi-\varepsilon$. Let $\psi_{n} \in D$ approximate $\psi$. Then, for large $n$.

$$
\phi_{n}(\varepsilon) \leq \psi_{n}
$$

Hence, by (S1)',

$$
S_{t} \phi_{n}(\varepsilon) \leq S_{t} \psi_{n} \quad \text { for large } n
$$

Therefore tending $n$ to $\infty$ we have

$$
S_{t}(\phi-\varepsilon) \leq S_{t} \psi
$$

On the other hand $\phi-\varepsilon$ converges to $\phi$, so (ii) implies $S_{t} \phi=\lim _{\varepsilon \downarrow 0} S_{t}(\phi-\varepsilon)$. Hence

$$
S_{t} \phi \leq S_{t} \psi
$$

(iii). For $\varepsilon>0$, we take $\psi \in D$ such that $\|\phi-\psi\|<\varepsilon$. Then we have

$$
\begin{gathered}
\left\|S_{t} \phi-S_{\theta} \phi\right\| \leq\left\|S_{t} \phi-S_{t} \psi\right\|+\left\|S_{t} \psi-S_{\theta} \psi\right\|+\left\|S_{\theta} \psi-S_{\theta} \phi\right\| \\
<2 \varepsilon+\left\|S_{t} \psi-S_{\theta} \psi\right\| \leq 2 \varepsilon+|t-\theta| K(\psi) .
\end{gathered}
$$

Hence there exists a small positive $\delta=\delta(\phi, \varepsilon)$ such that $\left\|S_{t} \phi-S_{\theta} \phi\right\|<3 \varepsilon$ whenever $i t-\theta \mid<\delta$.

(iv). By (S4)' we have $T_{t}{ }^{u} \phi_{n} \leq S_{t} \phi_{n}$ where $\phi_{n} \in D$ tends to $\phi$. Let- 
Non-Linear Semi-Group Attached to Stochastic Optimal Control 523 ting $n$ tend to $\infty$, we get (iv).

Proposition 4. $S_{t}$ is a semi-group on $L$.

Proof. Let $t$ and $\theta$ be binary, say $t=\frac{i}{2^{l}}$ and $\theta=\frac{j}{2^{l}}$. For $N \geq l$, we have

$$
\begin{aligned}
& S_{t+\theta}^{(N)} \phi=S_{\theta}{ }^{(N)}\left(S_{t}{ }^{(N)} \phi\right) \leq S_{\theta}{ }^{(N)}\left(S_{t} \phi\right), \\
& S_{\theta}\left(S_{t} \phi\right)=O_{i}-\lim _{N} S_{\theta}{ }^{(N)}\left(S_{t} \phi\right),
\end{aligned}
$$

and

$$
S_{\theta+t} \phi=O_{i}-\lim _{N} S_{\theta+t}^{(N)} \phi
$$

Hence

$$
S_{\theta+t} \phi \leq O_{i}-\lim _{N} S_{\theta}{ }^{(N)}\left(S_{t} \phi\right)=S_{\theta}\left(S_{t} \phi\right) .
$$

On the other hand, for $l \leq n \leq N$, we see

$$
S_{\theta}{ }^{(n)}\left(S_{t}{ }^{(N)} \phi\right) \leq S_{\theta}{ }^{(N)}\left(S_{t}{ }^{(N)} \phi\right)={S_{\theta+t}^{(N)}}^{(N)} \leq S_{\theta+t} \phi
$$

and recalling (J5) of Lemma 1 we have

$$
S_{\theta}^{(n)}\left(S_{t} \phi\right)=O_{i}-\lim _{N} S_{\theta}^{(n)}\left(S_{t}^{(N)} \phi\right)
$$

Therefore, for $n \geq l$,

$$
S_{\theta}^{(n)}\left(S_{t} \phi\right) \leq S_{\theta+t} \phi
$$

Tending $n$ to $\infty$, we get

$$
S_{\theta}\left(S_{t} \phi\right) \leq S_{\theta+t} \phi .
$$

From (15) and (16) we have

$$
S_{\theta}\left(S_{t} \phi\right)=S_{\theta+t} \phi \quad \text { for binary } t \text { and } \theta \text {. }
$$

Let $t_{n}$ be a binary approximation to $t$. Then for any binary $\theta$,

$$
S_{\theta}\left(S_{t_{n}} \phi\right)=S_{\theta+t_{n}} \phi .
$$

So appealing to (ii) and (iii) we get

$$
S_{\theta}\left(S_{t} \phi\right)=S_{\theta+t} \phi \quad \text { for binary } \theta .
$$


Again by virtue of (iii) we obtain the semi-group property of $S_{t}$.

Let $G$ be the generator of $S_{t}$, namely

$$
G \phi=\lim _{t \downarrow 0} \frac{1}{t}\left(S_{t} \phi-\phi\right)
$$

and

$$
D(G)=\left\{\phi \in L, \lim _{t \downarrow 0} \frac{1}{t}\left(S_{t} \phi-\phi\right) \text { exists }\right\}
$$

\section{Proposition 5.}

$$
G \phi=\sup _{u}\left(A^{u} \phi+f^{u}\right) \quad \text { for } \quad \phi \in D(G) \cap D .
$$

Moreover, if $f^{u} \in D\left(A^{u}\right)$ and $\sup _{u}\left\|A^{u} f^{u}\right\|<\infty$, then

$$
\begin{gathered}
D(G) \supset\left\{\phi \in D, A^{u} \phi \in D\left(A^{u}\right) \quad \text { for } \quad \forall u\right. \\
\text { and } \left.\sup _{u}\left\|A^{u}\left(A^{u} \phi\right)\right\|<\infty\right\}, \quad\left(\begin{array}{cl}
\text { say } & \Theta
\end{array}\right) .
\end{gathered}
$$

Proof. In the case $f^{u} \equiv 0$ for any $u$, we denote $S_{t}$ by $\Lambda_{t}$. Put $A \phi \equiv \sup _{u} G^{u} \phi=\sup _{u}\left(A^{u} \phi+f^{u}\right)$ and $\Delta=\frac{1}{2^{N}}$. Recalling (T5) we have for $\phi \in D$

$$
\begin{aligned}
S_{\Delta}{ }^{(N)} \phi-\phi & =\sup _{u}\left(T_{\Delta}{ }^{u} \phi-\phi\right)=\sup _{u} \int_{0}^{\Delta} P_{\theta}{ }^{u} G^{u} \phi d \theta \\
& \leq \sup _{u} \int_{0}^{\Delta} P_{\theta}{ }^{u} A \phi d \theta \leq \int_{0}^{\Delta} \Lambda_{\theta} A \phi d \theta
\end{aligned}
$$

Moreover

$$
\begin{aligned}
S_{2 \Delta}^{(N)} \phi & -S_{\Delta}{ }^{(N)} \phi=\sup _{u} T_{\Delta}{ }^{u}\left(S_{\Delta}{ }^{(N)} \phi\right)-\sup _{u} T_{\Delta}{ }^{u} \phi \\
& \leq \sup _{u}\left[T_{\Delta}{ }^{u}\left(S_{\Delta}{ }^{(N)} \phi\right)-T_{\Delta}{ }^{u} \phi\right]=\sup _{u}\left[P_{\Delta}{ }^{u}\left(S_{\Delta}{ }^{(N)} \phi\right)-P_{\Delta}{ }^{u} \phi\right] \\
& =\sup _{u}\left[P_{\Delta}{ }^{u}\left(S_{\Delta}{ }^{(N)} \phi-\phi\right)\right]=\Lambda_{\Delta}\left(S_{\Delta}{ }^{(N)} \phi-\phi\right) \\
& \leq \Lambda_{\Delta}\left(\int_{0}^{\Delta} \Lambda_{\theta} A \phi d \theta\right)=\int_{0}^{\Delta} \Lambda_{\Delta+\theta} A \phi d \theta=\int_{\Delta}^{2 \Delta} \Lambda_{\theta} A \phi d \theta .
\end{aligned}
$$

Suppose $S_{k \Delta}^{(N)} \phi-S_{(k-1) \Delta}^{(N)} \phi \leq \int_{(k-1) \Delta}^{k \Delta} \Lambda_{\theta} A \phi d \theta$. Then, by the similar calcula- 
Non-Linear Semi-Group Attachfo to Stochastic Optimal Control 525 tion, we see

$$
S_{(k+1) \Delta}^{(N)} \phi-S_{k \Delta}^{(N)} \phi \leq \Lambda_{\Delta}\left(S_{k \Delta}^{(N)} \phi-S_{(k-1) \Delta}^{(N)} \phi\right)<\int_{k \Delta}^{(k+1) \Delta} \Lambda_{\theta} A \phi d \theta .
$$

Hence taking the summation for $k$ we get

$$
S_{t}^{(N)} \phi-\phi \leq \int_{0}^{t} \Lambda_{\theta} A \phi d \theta \quad \text { for } \quad t=\frac{i}{2^{N}} .
$$

Tending $N$ to $\infty$ we have

$$
S_{t} \phi-\phi \leq \int_{0}^{t} \Lambda_{\theta} A \phi d \theta \text { for binary } t \text { and } \phi \in D .
$$

Since the both sides of (24) are continuous in $t$, (24) holds for any $t \geq 0$. Furthermore

$$
\frac{1}{t}\left(S_{t} \phi-\phi\right) \leq \frac{1}{t} \int_{0}^{t} \Lambda_{\theta} A \phi d \theta \leq\|A \phi\| 1,
$$

where 1 is the unit in $L$. On the other hand, by virtue of (T5) and (iv) of Proposition 3, we have

$$
\frac{1}{t}\left(S_{t} \phi-\phi\right) \geq \frac{1}{t}\left(T_{t}{ }^{u}-\phi\right)=\frac{1}{t} \int_{0}^{t} P_{\theta}{ }^{u} G^{u} \phi d \theta \geq-\left\|G^{u} \phi\right\| 1 .
$$

Therefore the set $\left\{\frac{1}{t}\left(S_{t} \phi-\phi\right), t>0\right\}$ is O-bounded. Hence $\inf _{\theta>0} \sup _{t>\theta} \frac{1}{t}$ $\times\left(S_{t} \phi-\phi\right)$, i.e. $O-\varlimsup_{t \downarrow 0} \frac{1}{t}\left(S_{t} \phi-\phi\right)$ exists, and $\sup _{\theta>0} \inf _{t>\theta} \frac{1}{t}\left(S_{t} \phi-\phi\right)$, i.e. $O-\frac{\lim }{t \downarrow 0} \frac{1}{t}\left(S_{t} \phi-\phi\right)$, exists. Since

$$
\lim _{t \downarrow 0} \frac{1}{t} \int_{0}^{t} \Lambda_{\theta} A \phi d \theta=A \phi
$$

and

$$
\lim _{t \downarrow 0} \frac{1}{t} \int_{0}^{t} P_{\theta}{ }^{u} G^{u} \phi d \theta=G^{u} \phi
$$

we have by (25), (26), (27) and (28),

$$
O-\varlimsup_{t \downarrow 0} \frac{1}{t}\left(S_{t} \phi-\phi\right) \leq A \phi
$$

and

$$
O-\lim _{t \downarrow 0} \frac{1}{t}\left(S_{t} \phi-\phi\right) \geq G^{u} \phi \quad \forall u .
$$


Hence

$$
O-\frac{\lim }{t \downarrow 0} \frac{1}{t}\left(S_{t} \phi-\phi\right) \geq \sup _{u} G^{u} \phi=A \phi .
$$

From (29) and (31) we have

$$
O-\varlimsup_{t \downarrow 0} \frac{1}{t}\left(S_{t} \phi-\phi\right)=O-\lim _{t \downarrow 0} \frac{1}{t}\left(S_{t} \phi-\phi\right)=A \phi .
$$

Thus, for $\phi \in D(G) \cap D$, we have

$$
G \phi=\lim _{t \downarrow 0} \frac{1}{t}\left(S_{t} \phi-\phi\right)=O-\lim _{t \downarrow 0} \frac{1}{t}\left(S_{t} \phi-\phi\right)=A \phi .
$$

Next we shall show (20). From (25)

$$
\frac{1}{t}\left(S_{t} \phi-\phi\right)-A \phi \leq \frac{1}{t} \int_{0}^{t} \Lambda_{\theta} A \phi d \theta-A \phi .
$$

By (27) the right side converges to 0 as $t \rightarrow 0$. Hence, for $\varepsilon>0$, there exists a positive $\delta=\delta(\varepsilon)$, such that

$$
\underset{x}{\text { ess. sup. }}\left[\frac{1}{t}\left(S_{t} \phi-\phi\right)(x)-A \phi(x)\right]<\varepsilon \quad \text { for } \quad t \in(0, \delta) \text {. }
$$

On the other hand, by (26) we have

$$
\begin{aligned}
& \frac{1}{t}\left(S_{t} \phi-\phi\right)-A \phi \geq \sup _{u} \frac{1}{t} \int_{0}^{t} P_{\theta}{ }^{u} G^{u} \phi d \theta-A \phi \\
& =\sup _{u} \frac{1}{t} \int_{0}^{t} P_{\theta}{ }^{u} G^{u} \phi d \theta-\sup _{u} G^{u} \phi \geq \inf _{u}\left[\frac{1}{t} \int_{0}^{t} P_{\theta}{ }^{u} G^{u} \phi d \theta-G^{u} \phi\right] .
\end{aligned}
$$

For $\phi \in \Theta$, we have $G^{u} \phi \in D\left(A^{u}\right)$ and

$$
P_{\theta}^{u} G^{u} \phi-G^{u} \phi=\int_{0}^{\theta} P_{s}^{u} A^{u} G^{u} \phi d s .
$$

Thus

$$
\frac{1}{t} \int_{0}^{t} P_{\theta}^{u} G^{u} \phi d \theta-G^{u} \phi=\frac{1}{t} \int_{0}^{t}\left(\int_{0}^{\theta} P_{s}^{u} A^{u} G^{u} \phi d s\right) d \theta
$$

So we have

$$
\left\|\frac{1}{t} \int_{0}^{t} P_{\theta}^{u} G^{u} \phi d \theta-G^{u} \phi\right\| \leq\left\|A^{u} G^{u} \phi\right\| t \leq\left\|A^{u}\left(A^{u} \phi\right)+A^{u} f^{u}\right\| t .
$$

Therefore by (33) and (34) we have 
Non-Linear Semi-Group AtTached to Stochastic Optimal Control 527

$$
\underset{x}{\operatorname{ess.inf}}\left[\frac{1}{t}\left(S_{t} \phi-\phi\right)-A \phi\right] \geq-\sup _{u}\left\|A^{u}\left(A^{u} \phi\right)+A^{u} f^{u}\right\| t
$$

Hence (32) and (35) complete the proof of (20).

Remark 1. If $S_{t} \phi$ is differentiable in $t>0$ and $S_{t} \phi$ belongs to $D$, then

$$
\left\{\begin{array}{l}
\frac{d}{d t} S_{t} \phi=\sup _{u}\left(A^{u} S_{t} \phi+f^{u}\right), t>0, \\
S_{0} \phi=\phi .
\end{array}\right.
$$

This is the so-called Bellman equation. So $S_{t}$ is called a Bellman semigroup.

Remark 2. If each $A^{u}$ is a bounded operator on $L$ and

$$
\sup _{u}\left\|A^{u}\right\|<\infty,
$$

then $\sup _{u}\left\|A^{u} f^{u}\right\|<\infty$ and $\Theta=L$. Moreover $S_{t} \phi$ is differentiable in $t$ and satisfies the Bellman equation.

Proof. Since $A^{u}$ is a bounded linear operator on $L$,

$$
P_{t}^{u}=\sum_{k=0}^{\infty} \frac{1}{k !}\left(t A^{u}\right)^{k}=\exp t A^{u}
$$

and $D\left(A^{u}\right)=L$. Hence $f^{u} \in D\left(A^{u}\right)$ and $\sup _{u}\left\|A^{u} f^{u}\right\| \leq \sup \left\|A^{u}\right\| h<\infty$. Moreover $\sup _{u}\left\|A^{u} \phi\right\|<\infty$, for any $\phi \in L$. Thus $D=L$. Since $\sup _{u}\left\|A^{u}\left(A^{u} \phi\right)\right\|$ $\leq\left(\sup \left\|A^{u}\right\|\right)^{2}\|\phi\|$, we have $\Theta=L$.

For the proof of the latter half, we apply the same method as for linear semi-groups. Since $D(G) \supset \Theta=L$, the right derivative of $S_{t} \phi$,

$$
\frac{d^{+}}{d t} S_{t} \phi=\lim _{\theta \downarrow 0} \frac{1}{\theta}\left(S_{t+\theta} \phi-S_{t} \phi\right)
$$

exists and, by $\Theta=L$,

$$
\frac{d^{+}}{d t} S_{t} \phi=\sup _{u}\left(A^{u} S_{t} \phi+f^{u}\right)=A S_{t} \phi
$$

Hence, for any $F \in L^{\prime}$, we have 


$$
F\left(A S_{t} \phi\right)=F\left(\frac{d^{+}}{d t} S_{t} \phi\right)=\lim _{\theta \downarrow 0} \frac{1}{\theta}\left(F\left(S_{t+\theta} \phi\right)-F\left(S_{t} \phi\right)\right)=\frac{d^{+}}{d t} F\left(S_{t} \phi\right)
$$

On the other hand by (36) we get

$$
\begin{aligned}
\left\|A S_{t} \phi-A S_{\theta} \phi\right\| \leq \sup _{u}\left\|A^{u} S_{t} \phi-A^{u} S_{\theta} \phi\right\| \\
=\sup _{u}\left\|A^{u}\left(S_{t} \phi-S_{\theta} \phi\right)\right\| \leq\left(\sup _{u}\left\|A^{u}\right\|\right)\left\|S_{t} \phi-S_{\theta} \phi\right\| .
\end{aligned}
$$

Therefore $A S_{t} \phi$ is continuous in $t$. So $F\left(A S_{t} \phi\right)$ is a real continuous function of $t$, namely the right derivative of $F\left(S_{t} \phi\right)$ is continuous. Therefore $F\left(S_{t} \phi\right)$ is differentiable and its derivative $\frac{d F\left(S_{t} \phi\right)}{d t}$ is continuous. Therefore

$$
\begin{aligned}
F\left(S_{t} \phi-\phi\right) & =F\left(S_{t} \phi\right)-F(\phi)=\int_{0}^{t} \frac{d}{d \theta} F\left(S_{\theta} \phi\right) d \theta \\
& =\int_{0}^{t} F\left(A S_{\theta} \phi\right) d \theta=F\left(\int_{0}^{t} A S_{\theta} \phi d \theta\right) .
\end{aligned}
$$

Since $F$ is arbitrary, (37) implies

$$
S_{t} \phi-\phi=\int_{0}^{t} A S_{\theta} \phi d \theta \text {. }
$$

By the continuity of $A S_{\theta} \phi$, (38) implies the differentiability of $S_{t} \phi$. Therefore by Remark $1 S_{t} \phi$ satisfies the Bellman equation. In fact the operator $S_{t}$ thus obtained is identical with $e^{t A}$ in the sense of [1].

Proposition 6. If $\widetilde{S}_{t}$ is a semi-group on $L$ satisfying the condition (i) $\sim(\mathrm{iv})$, then for any $t \geq 0$ and $\phi \in L$,

$$
S_{t} \phi \leq \widetilde{S}_{t} \phi
$$

Proof. Putting $\Delta=\frac{1}{2^{N}}$, we have

$$
S_{\Delta}{ }^{(N)} \phi=\sup _{u} T_{\Delta}{ }^{u} \phi \leq \widetilde{S}_{\Delta} \phi, \quad \forall \phi \in L .
$$

Suppose

$$
S_{k \Delta}^{(N)} \phi \leq \widetilde{S}_{k \Delta} \phi
$$

Then

$$
S_{(k+1) \Delta}^{(N)} \phi=S_{\Delta}^{(N)}\left(S_{k \Delta}^{(N)} \phi\right) \leq S_{\Delta}^{(N)}\left(\widetilde{S}_{k \Delta} \phi\right) \leq \widetilde{S}_{\Delta}\left(\widetilde{S}_{k \Delta} \phi\right)=\widetilde{S}_{(k+1) \Delta} \phi
$$


Non-Linear Semi-Group Attached to Stochastic Optimal Control 529 Hence, for any $k$, we have (40).

This implies for any binary $t$

$$
S_{t}^{(n)} \phi \leq \widetilde{S}_{t} \phi \quad \text { for large } n .
$$

Therefore for binary $t$

$$
S_{t} \phi=O_{i}-\lim _{n} S_{t}^{\left({ }^{n}\right)} \phi \leq \widetilde{S}_{t} \phi
$$

Since the both sides are continuous in $t$, we complete the proof of Proposition 6 .

For any constant $c \geq 0$, we replace $P_{t}^{u}$ by $e^{-c t} P_{t}^{u}$. Then we can easily show the following,

Corollary. Theorem 1 is still valid, when we replace (iv) and (5) respectively by

$$
e^{-c t} P_{t}^{u}+\int_{0}^{t} e^{-c \theta} P_{\theta}^{u} \phi d \theta \leq S_{t} \phi
$$

and

$(\mathrm{v})^{\prime} \quad G \phi=\sup _{u}\left(A^{u} \phi-c \phi+f^{u}\right), \quad$ for $\quad \phi \in D(G) \cap D$.

For positive $c$, we denote the semi-group of Corollary by $\widetilde{S}_{t}$.

Proposition 7. There exists a unique $v \in L$ such that

$$
\lim _{t \uparrow \infty} \widetilde{S}_{t} \phi=v \quad \text { for any } \phi \in L .
$$

Proof. Using $e^{-c t} P_{t}{ }^{u}$ instead of $P_{t}^{u}$, we define $\tilde{J}(N)$ and $\widetilde{S}^{(N)}$ by the similar way. Then, putting $\tilde{J}=\tilde{J}(N)$ and $\Delta=\frac{1}{2^{N}}$, we have

$$
\|\tilde{J} \phi-\tilde{J} \psi\| \leq \sup _{u}\left\|e^{-c \Delta} P_{\Delta}{ }^{u} \phi-e^{-c \Delta} P_{\Delta} \psi\right\| \leq e^{-c \Delta}\|\phi-\psi\| .
$$

Moreover we can show (41) by the induction,

$$
\left\|\tilde{J}^{k} \phi-\tilde{J}^{k} \psi\right\| \leq e^{-c k s}\|\phi-\psi\| .
$$

On the other hand we can easily see the following inequality 


$$
\|\tilde{J} \phi\| \leq e^{-c \Delta}\|\phi\|+\frac{h}{c}\left(1-e^{-c \Delta}\right)
$$

and moreover, we have (42) by the induction,

$$
\left\|\tilde{J}^{k} \phi\right\| \leq e^{-c k s}\|\phi\|+\frac{h}{c}\left(1-e^{-c k \Delta}\right) .
$$

(41) and (42) mean, for $t=\frac{k}{2^{N}}$,

$$
\left\|\widetilde{S}_{t}^{(N)} \phi-\widetilde{S}_{t}^{(N)} \psi\right\| \leq e^{-c t}\|\phi-\psi\|
$$

and

$$
\left\|{\widetilde{S_{t}}}^{(N)} \phi\right\| \leq e^{-c t}\|\phi\|+\frac{h}{c}\left(1-e^{-c t}\right)
$$

Therefore, for binary $t$, we have (43) and (44),

$$
\left\|\widetilde{S}_{t} \phi-\widetilde{S}_{t} \psi\right\| \leq \lim _{N}\left\|\widetilde{S}_{t}^{(N)} \phi-\widetilde{S}_{t}^{(N)} \psi\right\| \leq e^{-c t}\|\phi-\psi\|
$$

and

$$
\left\|\widetilde{S}_{t} \phi\right\| \leq e^{-c t}\|\phi\|+\frac{h}{c}\left(1-e^{-c t}\right) \leq\|\phi\|+\frac{h}{c}
$$

Since the both sides of the above inequalities (43) and (44) are continuous in $t$, we have

$$
\left\|\widetilde{S}_{t+\theta} \phi-\widetilde{S}_{t} \phi\right\|=\left\|\widetilde{S}_{t}\left(\widetilde{S}_{\theta} \phi\right)-\widetilde{S}_{t} \phi\right\| \leq e^{-c t}\left(2\|\phi\|+\frac{h}{c}\right) .
$$

Hence there exists $\lim _{t \rightarrow \infty} \widetilde{S}_{t} \phi$, say $\boldsymbol{v}_{\phi}$. By virtue of (43), we can see that $v_{\phi}$ does not depend on $\phi$.

Corollary. $\widetilde{S}_{t} v=v$ for any $t \geq 0$, and if $v$ belongs to $D$, then $\sup _{u}\left(A^{u} v-c v+f^{u}\right)=0$.

\section{§ 4. Proof of Theorem 2}

For any $\lambda \geq 0$ and $g \in L$ we define

$$
T_{t}^{u \lambda g} \phi=e^{-\lambda t} P_{t}^{u} \phi+\int_{0}^{t} e^{-\lambda \theta} P_{\theta}^{u}\left(f^{u}+\lambda g\right) d \theta .
$$

Then we have

$$
\left\|T_{t}^{u \lambda g} \phi\right\| \leq e^{-(\lambda+c) t}\|\phi\|+\frac{h}{c}\left(1-e^{-c t}\right)+\left(1-e^{-\lambda t}\right)\|g\|
$$


Non-Linear Semi-Group Attached to Stochastic Optimal Control 531 and its generator $G^{u \lambda g}$ is as follows

$$
G^{u \lambda g} \phi=A^{u} \phi-\lambda \phi+f^{u}+\lambda g \text {. }
$$

For simplicity we omit $g$ in $T_{t}^{u \lambda g}$ and $G^{u \lambda g}$ for the moment, if any confusion does not occur. In order to prove Theorem 2, we apply the same method as [4], namely we take $\Gamma \times[0, \infty)$ for the control region. Appealing to (2), we can define $J=J(N)$ by

$$
J \phi=\sup _{u \lambda} T_{1 / 2 N}^{u \lambda} \phi, \phi \in L
$$

and

$$
J^{k+1} \phi=J\left(J^{k} \phi\right), J^{0} \phi=\phi .
$$

Then Lemma 1 is easy.

Lemma 1. Putting $\Delta=\frac{1}{2^{N}}$, we have

(J0) $J^{k+l} \phi=J^{k}\left(J^{l} \phi\right)=J^{l}\left(J^{k} \phi\right)$,

(J1) $J^{k} \phi \leq J^{k} \psi$ whenever $\phi \leq \psi$,

(J2) $\left\|J^{k} \phi-J^{k} \psi\right\| \leq e^{-c k s}\|\phi-\psi\|$,

(J3) $\left\|J^{k} \phi\right\| \leq e^{-c k s}\|\phi\|+\frac{h}{c}\left(1-e^{-c k s}\right)+\|g\|$,

(J4) $\phi=O_{i}-\lim _{n} \phi_{n}$ implies $J^{k} \phi=O_{i}-\lim _{n} J^{k} \phi_{n}$.

(J5) $g \leq J \phi$.

Proof. We show (J3) by the induction. For $k=1$, (J3) comes from (2). Suppose (J3) holds for $k$. Then we have

$$
\left\|J^{k+1} \phi\right\|=\left\|J\left(J^{k} \phi\right)\right\| \leq \sup _{u \lambda}\left\|T_{\Delta}{ }^{u \lambda}\left(J^{k} \phi\right)\right\| .
$$

Recalling (2) we see

$$
\begin{aligned}
& \left\|T_{\Delta}^{u \lambda}\left(J^{k} \phi\right)\right\| \leq e^{-(\lambda+c) \Delta}\left\|J^{k} \phi\right\|+\frac{h}{c}\left(1-e^{-c \Delta}\right)+\left(1-e^{-\lambda \Delta}\right)\|g\| \\
& \leq e^{-(\lambda+c) \Delta}\left(e^{-c k s}\|\phi\|+\frac{h}{c}\left(1-e^{-c k \Delta}\right)+\|g\|\right)+\frac{h}{c}\left(1-e^{-c \Delta}\right)+\left(1-e^{-\lambda \Delta}\right)\|g\| \\
& \leq e^{-c(k+1) \Delta}\|\phi\|+\frac{h}{c}\left(e^{-c \Delta}-e^{-c(k+1) \Delta}+1-e^{-c \Delta}\right)+\|g\| .
\end{aligned}
$$

From (3) and (4) we have (J3) for $k+1$.

We have, for any $u \in \Gamma$ and $t>0$, 


$$
g=\lim _{\lambda \rightarrow \infty} T_{t}^{u \lambda} \phi
$$

Hence (J5) is valid.

Define $\boldsymbol{S}_{t}{ }^{(N)}$ by $\boldsymbol{S}_{t}{ }^{(N)} \phi=J^{k}(N) \phi$ for $t=\frac{k}{2^{N}}$. Then $\boldsymbol{S}_{t}{ }^{(N)} \phi$ is increasing as $N \rightarrow \infty$. Moreover we have

Lemma 2. If $\phi \leq g$, then $\boldsymbol{S}_{t}^{(N)} \phi$ is increasing as $t \rightarrow \infty$.

Proof. Putting $\Delta=1 / 2^{N}$, we get by (J5)

$$
\phi \leq g \leq \boldsymbol{S}_{4}{ }^{(N)} \phi .
$$

Hence, by (J1),

$$
\phi \leq \boldsymbol{S}_{\Delta}^{(N)} \phi \leq \boldsymbol{S}_{2 \Delta}^{(N)} \phi \leq \cdots \boldsymbol{S}_{k \Delta}^{(N)} \phi \leq \boldsymbol{S}_{(k+1)}^{(N)} \phi .
$$

(J3) means the following (7).

$$
\left\|\boldsymbol{S}_{t}^{(N)} \phi\right\| \leq e^{-c t}\|\phi\|+\frac{h}{c}\left(1-e^{-c t}\right)+\|g\| .
$$

Therefore, for binary $t$, the set $\left\{\boldsymbol{S}_{t}{ }^{(N)} \phi, N\right.$ large $\}$ if $O$-bounded. Hence we can define $\boldsymbol{S}_{t}$ by

$$
S_{t} \phi=O_{i}-\lim _{N} \boldsymbol{S}_{t}^{(N)} \phi \text { for binary } t .
$$

From (J4) we can again see, for binary $t$,

$$
S_{t} \phi=O_{i}-\lim _{n} S_{t} \phi_{n} \quad \text { if } \quad \phi=O_{i}-\lim _{n} \phi_{n} .
$$

Therefore we can derive the semi-group property on binary parameter.

$$
\boldsymbol{S}_{t+\theta} \phi=\boldsymbol{S}_{t}\left(\boldsymbol{S}_{\theta} \phi\right)=\boldsymbol{S}_{\theta}\left(\boldsymbol{S}_{t} \phi\right) \text { for binary } t \text { and } \theta \text {. }
$$

Again, by (7), we have

$$
\left\|\boldsymbol{S}_{t} \phi\right\| \leq e^{-c t}\|\phi\|+\frac{h}{c}\left(1-e^{-c t}\right)+\|g\|
$$

Hence the set $\left\{\boldsymbol{S}_{t} \phi\right.$, binary $\left.t\right\}$ is also $O$-bounded.

Lemma 3. If $\phi \leq g$, then $\boldsymbol{S}_{t} \phi$ is increasing in $t$ and $O_{i}-\lim _{t} S_{t} \phi$ exists, say $v_{\phi}$. Moreover 
Non-Linear Semi-Group Attached to Stochastic Optimal Control 533

$$
g \leq v_{\phi}
$$

Proof. By Lemma 2 we have for $t<\theta$,

$$
\boldsymbol{S}_{t} \phi=O_{i}-\lim _{N} \boldsymbol{S}_{t}^{(N)} \phi \leq O_{i}-\lim _{N} \boldsymbol{S}_{\theta}{ }^{(N)} \phi=\boldsymbol{S}_{\theta} \phi
$$

Hence $S_{t} \phi$ is increasing as binary $t \rightarrow \infty$. (10) is clear by (J5). For simplicity we put $v=v_{\phi}$ if any confusion does not occur.

Lemma 4. $v$ is $\boldsymbol{S}_{t}$-invariance, i.e.

$$
S_{t} v=v \text { for binary } t \text {. }
$$

Proof. By the definition of $v$ and (8),

$$
\boldsymbol{S}_{t} \boldsymbol{v}=\boldsymbol{S}_{t}\left(O_{i}-\lim _{\theta} \boldsymbol{S}_{\theta} \phi\right)=O_{i}-\lim _{\theta} \boldsymbol{S}_{t+\theta} \phi=v
$$

Proposition 8. $v$ is an $S_{t}$-excessive majorant of $g$, i.e. $v \geq g$ and (12)

$$
S_{t} v \leq v, \forall t \geq 0
$$

Proof. By the definitions of $S_{t}$ and $S_{t}$, we have

$$
S_{t} \psi \leq S_{t} \psi \quad \forall \text { binary } t \text { and } \psi \in L .
$$

Hence by Lemma 4

$$
S_{t} \boldsymbol{v} \leq \mathbf{S}_{t} v=v
$$

Namely we get (12) for binary $t$. Since $S_{t} v$ is continuous in $t$, (12) is valid for any $t$. Recalling (10) we complete the proof.

Proposition 9. For any $\phi \leq g, v_{\phi}$ is the least $S_{t}$-excessive majorant of $g$.

Proof. Let $V$ be an $S_{t}$-excessive majorant of $g$. Recalling the definitions of $T_{t}{ }^{u \lambda V}$ and $T_{t}{ }^{u}$, we have

$$
T_{t}^{u \lambda v} \psi=e^{-\lambda t} P_{t}^{u} \psi+\int_{0}^{t} e^{-\lambda \theta} P_{\theta}^{u}\left(f^{u}+\lambda V\right) d \theta
$$


and

$$
T_{t}^{u 00} \psi=P_{t}^{u} \psi+\int_{0}^{t} P_{\theta}^{u} f^{u}=T_{t}^{u} \psi
$$

Hence

$$
\begin{aligned}
T_{t}{ }^{u \lambda V} V=e^{-\lambda t} T_{t}{ }^{u} V & +\int_{0}^{t} e^{-\lambda \theta} P_{\theta}{ }^{u} f^{u} d \theta \\
& +\lambda \int_{0}^{t} e^{-\lambda \theta} P_{\theta}{ }^{u} g d \theta-e^{-\lambda t} \int_{0}^{t} P_{\theta}{ }^{u} f^{u} d \theta,
\end{aligned}
$$

and, from (14), we see

$$
\begin{aligned}
\lambda \int_{0}^{t} e^{-\lambda \theta} P_{\theta}{ }^{u} V d \theta & =\lambda \int_{0}^{t} e^{-\lambda \theta}\left(T_{\theta}{ }^{u} V-\int_{0}^{\theta} P_{s}^{u} f^{u} d s\right) d \theta \\
& =\lambda \int_{0}^{t} e^{-\lambda \theta} T_{\theta}{ }^{u} V d \theta-\int_{0}^{t}\left(e^{-\lambda s}-e^{-\lambda t}\right) P_{s}^{u} f^{u} d s .
\end{aligned}
$$

Therefore, by (15) and (16) we have

$$
T_{t}{ }^{u \lambda V} V=e^{-\lambda t} T_{t}{ }^{u} V+\lambda \int_{0}^{t} e^{-\lambda \theta} T_{\theta}{ }^{u} V d \theta
$$

Since " $T_{t}{ }^{u} \psi \leq S_{t} \psi$ " and $V$ is $S_{t}$-excessive, we have

$$
e^{-\lambda t} T_{t}{ }^{u} V \leq e^{-\lambda t} S_{t} V \leq e^{-\lambda t} V .
$$

Combining (18) with (17) we can see

$$
T_{t}^{u \lambda V} V \leq e^{-\lambda t} V+\lambda \int_{0}^{t} e^{-\lambda \theta} V d \theta=V .
$$

Hence we have, denoting $J(N)$ for $T_{t}^{u \lambda v}$ by $\tilde{J}(N)$,

$$
\tilde{J}(N) V \leq V \quad \text { and } \quad \tilde{J}^{k}(N) V \leq V .
$$

This tells us the following inequality,

$$
\tilde{\boldsymbol{S}}_{t} V \leq V \text { for binary } t .
$$

Appealing to " $g \leq V$ " and the definition of $T_{t}{ }^{u \lambda g}$, we have

$$
T_{t}^{u \lambda g} \psi \leq T_{t}^{u \lambda v} \psi \quad \forall \psi \in L
$$

Hence

$$
J(N) \psi \leq \tilde{J}(N) \psi \quad \text { and } \quad \boldsymbol{S}_{t} \psi \leq \widetilde{\boldsymbol{S}}_{t} \psi
$$

So, by (21), we have for binary $t$, 
Non-Linear Semi-Group Attached to Stochastic Optimal Control 535

$$
\boldsymbol{S}_{t} \phi \leq \boldsymbol{S}_{t} g \leq \boldsymbol{S}_{t} V \leq \tilde{\boldsymbol{S}}_{t} V \leq V
$$

Tending $t$ to $\infty$, we can derive

$$
v \leq V \text {. }
$$

Corollary. $v_{\phi}=v_{g} \forall \phi \leq g$.

Proof. Since the least $S_{t}$-excessive majorant of $g$ is unique, $v_{\phi}=v_{g}$.

\section{§ 5. Examples}

We will show two simple examples of control problems related Markov processes with exponential holding times, [cf. 5].

Example 1. Let $A^{u}=\left(a^{u}(i, j)\right)$ be an $l \times l$-matrix. Suppose $a^{u}(i, j) \geq 0$ for $i \neq j$ and $\sum_{j=1}^{l} a^{u}(i, j)=0$. Then $A^{u}$ is the generator of the transition semi-group $P_{t}^{u}=\left(P_{t}^{u}(i, j)\right)=e^{t A}$.

Put $\mu\{i\}=1, i=1, \cdots, l$ and $\mu\left(R^{1}-\{1,2, \cdots, l\}\right)=0$. Then $A^{u}$ becomes a bounded linear operator on $L=L_{\infty}\left(R^{1}, \mu\right)$ and $P_{t}{ }^{u}$ a positive contractive and continuous semi-group on $L$. Assume

$$
\sup _{u}\left|a^{u}(i, j)\right|<\infty, \quad \forall i j=1, \cdots, l .
$$

Thus $\sup _{u}\left\|A^{u}\right\|<\infty$. Let $\sup _{u}\left|f^{u}(i)\right|<\infty$ for $i=1, \cdots, l$. Then we can construct Bellman semi-group $S_{t}$ for $\left\{A^{u}, f^{u}\right\}$. Moreover, for $\phi \in L, S_{t} \phi$ is a solution of the following Bellman equation,

$$
\left\{\begin{array}{l}
\frac{d S_{t} \phi(i)}{d t}=\sup _{u}\left[\sum_{j=1}^{l} a^{u}(i, j) S_{t} \phi(j)+f^{u}(i)\right], i=1, \cdots, l, \\
S_{0} \phi(i)=\phi(i) .
\end{array}\right.
$$

Example 2. Let $X^{u}$ be a 1-dimensional Lévy process of pure jump type with finite Lévy measure $n^{u}$

$$
X(t)=x+\int_{R^{1}} \int_{0}^{t} z N^{u}(d s d z)
$$

and $E N^{u}(d s d z)=d s n^{u}(d z)$. Thus every point of $R^{1}$ is an exponential 
holding point.

Suppose that $n^{u}$ has the density, say $n^{u}(d z)=n^{u}(z) d z$. Put $Y^{u}(t)$ $=\int_{R^{1}} \int_{0}^{t} z N^{u}(d s d z)$. We denote its $i$-th jump time by $\tau_{i}{ }^{u}, \tau_{0}{ }^{u}=0$, and $Y^{u}\left(\tau_{i}{ }^{u}\right)-Y^{u}\left(\tau_{i-1}^{u}\right)$ by $\zeta_{i}{ }^{u}$. For simplicity we skip the suffix $u$ if any confusion does not occur. We have the following well-known facts,

(i) $\tau_{i}-\tau_{i-1}, i=1,2, \cdots, \zeta_{i}, i=1,2, \cdots$ are independent.

(ii) $P\left(\tau_{i}-\tau_{i-1}>t\right)=e^{-t \lambda}$ where $\lambda=n\left(R^{1}\right)$.

(iii) $P\left(\zeta_{i} \in A\right)=\frac{n(A)}{\lambda}=\frac{1}{\lambda} \int_{A} n(z) d z$.

Hence

(2) $P(Y(t) \in A)=\chi_{A}(O) P\left(\tau_{1}<t\right)+\sum_{i=1}^{\infty} P\left(Y\left(\tau_{i}\right) \in A\right) P\left(\tau_{i} \leq t<\tau_{i+1}\right)$

$$
\begin{aligned}
& =\chi_{A}(O) e^{-t \lambda}+\sum_{i=1}^{\infty} P\left(\zeta_{1}+\cdots+\zeta_{i} \in A\right) P\left(\tau_{i} \leq t \leq \tau_{i+1}\right) \\
& =\chi_{A}(O) e^{-t \lambda}+m(A, t) .
\end{aligned}
$$

By virtue of (i) and (iii) the measure $m(\cdot, t)$ is absolutely continuous w.r. to the Lebesgue measure $\mu$. Suppose $\phi=\psi \mu$-a.e. Then, for any $x$ where " $\psi(x)=\phi(x)$ " holds, we see

$$
\begin{aligned}
P_{t} \phi(x) & =E_{x} \phi(X(t))=E \phi(x+Y(t)) \\
& =\phi(x) e^{-\lambda t}+\int \phi(x+y) m^{\prime}(y, t) d y \\
& =\phi(x) e^{-\lambda t}+\int \phi(y) m^{\prime}(y-x, t) d y \\
& =\psi(x) e^{-\lambda t}+\int \psi(y) m^{\prime}(y-x, t) d y=P_{t} \psi(x) .
\end{aligned}
$$

Hence the transition semi-group $P_{t}$ can act on $L=L_{\infty}\left(R^{1}, \mu\right)$. On the other hand we have

$$
\begin{aligned}
\left|P_{t} \phi(x)-\phi(x)\right| & \leq|\phi(x)|\left(1-e^{-t \lambda}\right)+\|\phi\| P\left(\tau_{1} \leq t\right) \\
& \leq 2\|\phi\|\left(1-e^{-t \lambda}\right) \rightarrow 0 \quad \text { as } \quad t \downarrow 0 .
\end{aligned}
$$

So $P_{t}$ is strongly continuous.

Thus $P_{t}^{u}$ is a positive contractive and strongly continuous linear semigroup on $L$ whose generator $A^{u}$ is 
Non-Linear Semi-Group Attached to Stochastic Optimal Control 537

$$
A^{u} \phi(x)=\int_{R^{1}}(\phi(x+y)-\phi(x)) n^{u}(y) d y, \quad \phi \in L .
$$

Since $\left\|A^{u} \phi\right\| \leq 2\|\phi\| \lambda^{u}$, this example 2 satisfies the condition (36) of Re$\operatorname{mark} 2$, if

$$
\sup _{u} \lambda^{u}<\infty
$$

Therefore, for $f^{u} \in L$ with (A3), we have a solution, $V(t, x)=S_{t} \phi(x)$, of Bellman equation

$$
\left\{\begin{array}{l}
\frac{\partial V(t, x)}{\partial t}=\sup _{u}\left[\int_{R^{1}}(V(t, x+y)-V(t, x)) n^{u}(y) d y+f^{u}(x)\right] \text { a.e. } \forall t>0, \\
V(0, x)=\phi(x) .
\end{array}\right.
$$

\section{References}

[1] Crandall, M. G. and Liggett, T. M., Generation of semi-groups of non-linear transformations on general Banach spaces, Amer. J. Math., 18 (1971), 265-298.

[2] Fleming, W. H. and Rishel, R. W., Deterministic and stochastic optimal control, Appl. of Math., 1, Springer-Verlag, 1975.

[3] Grigelionis, B. I. and Shiryaev, A. N., On Stefan problem and optimal stopping rules for Markov processes, Theor. Probability Appl., 11 (1966), 541-558.

[4] Krylov, N. V., Control of a solution of a stochastic integral equation, Theor. Probability Appl., 17 (1972), 114-131.

[5] Itô, K., Stochastic Processes, Lect. Notes, 16, Aarhus Univ. 1969.

[6] Nisio, M., Some remarks on stochastic optimal controls, 3rd USSR-Japan Symp. Probability Theory, 1975.

[7] Yosida, K., Functional analysis, Springer-Verlag, 1968. 
